\title{
Pulse shape simulations for organic scintillation detectors using Geant4
}

\author{
Caroline Holroyd ${ }^{1}$, Michael Aspinall ${ }^{1}$, and Tom Deakin ${ }^{2}$ \\ ${ }^{1}$ Lancaster University, United Kingdom \\ ${ }^{2}$ LabLogic Systems Ltd., United Kingdom \\ c.holroyd2@lancaster.ac.uk
}

\begin{abstract}
The accurate simulation of the temporal pulse shapes from organic scintillation detectors capable of pulse shape discrimination (PSD) presents the opportunity to assess the pulse shape discrimination of these detectors prior to fabrication. The aim of this study is the simulation of the temporal pulse shapes from EJ-276, a PSD-capable plastic scintillator developed by Eljen Technologies. PSD plastic scintillators are increasingly replacing organic liquid scintillators for the detection of neutrons in the presence of mixed radiation fields for nuclear security applications. Plastics are inexpensive, robust and can be fabricated in a variety of shapes and sizes. They offer a solid-state alternative to liquid scintillators which can be difficult to transport due to the risk of leakage. However, the PSD performance of plastic scintillators has been observed to decrease due to various factors which combine to influence the overall shape of the pulse. The Monte Carlo toolkit Geant4 has been used to simulate the temporal pulse shapes from an EJ-276 plastic scintillator coupled to a photomultiplier tube (PMT). All three decay time components of EJ-276 have been modelled, utilising new methods available in the latest version of Geant4, for two different scintillator geometries. The simulated $n / \gamma$ pulse shapes reproduce the features important for PSD. Future work will involve integrating the temporal response of the PMT with existing pulse shape simulations. Simulated data will then be compared with experimental measurements.
\end{abstract}

Keywords -Monte Carlo simulations, Geant4, plastic scintillators, pulse shape discrimination

\section{INTRODUCTION}

$\mathrm{T}$ HE temporal shape of the output pulse formed following the interaction of ionising radiation with a scintillation detector enables characterisation of the incident radiation. The pulses derived from organic and inorganic scintillators are typically represented by an exponential rise time and multiple decay time components. These time components relate to the scintillation mechanism of the material and can differ with the type of interacting particle. This allows information on the properties of the incident radiation to be extracted from the pulse shape using pulse shape analysis techniques.

Pulse shape discrimination (PSD) is a pulse shape analysis technique used for the separation of neutron and gamma ray induced signals. Signal separation is achieved by exploiting the subtle differences in the shapes of the pulses formed following neutron and gamma ray interactions in a scintillator material. Pulse shape discrimination (PSD) using organic scintillators utilises the fact that organic material with certain molecular properties emit scintillation light with two decay components, referred to as the fast and slow components. The fast component is emitted a few nanoseconds following particle interaction, while the slow component is emitted a few hundred nanoseconds after. Various techniques have been developed for PSD with the aim of achieving the greatest separation of neutron and gamma ray induced signals.

One of the most commonly used methods for PSD with organic scintillators in a mixed radiation field is based on analysis of the decay tail of the pulse [1]. A distinct difference is observed in the decay tail for neutron and gamma ray induced pulses. This is because neutrons experience a greater rate of energy loss in the scintillator compared to gamma rays, resulting in an enhanced slow component for neutron interactions. This is observable in the decay tails of the pulse shapes and signal separation is achieved on this basis.

The aim of this study is the accurate simulation of the temporal pulse shapes from an EJ-276 PSD-capable plastic scintillation detector using the Monte Carlo simulation toolkit Geant4. This has the potential to enable the PSD performance of organic scintillation detectors to be assessed in the initial stages of detector design. Factors which reduce the performance can then be identified and their effects mitigated with the aim of optimising detector performance. This is important for plastic scintillation detectors since the PSD performance of these detectors can be unreliable and is known to fluctuate as a result of numerous factors which combine to influence the shape of the output pulse. One such factor is the geometry of the scintillator, where the ability to separate neutron and gamma ray induced signals is observed to degrade as the size of the scintillator is increased [2]. Other factors include reflector material, photodetector position and location of particle interaction in the scintillator [3].

Geant4 is widely used for the simulation of plastic scintillation detectors. However, simulations of the temporal pulse shapes from PSD-capable plastic scintillators have previously neglected to model all known decay time components associated with the scintillator and their dependency on particle type. This has impacted on the overall 
accuracy of pulses simulated using Geant 4 when compared with experimental measurements. This was identified as a limitation of Geant4 for simulating the pulse shapes of scintillation detectors with more than two decay time components [4]. This includes the PSD-capable plastic scintillator EJ-276 (previously EJ-299-33) which is stated by the manufacturer to have three particle-dependent decay time components. Prior to Geant 4 version 10.7 the number of decay time components was limited. For particle-independent yields two decay time components could be specified, while for particle-dependent yields this was reduced to one [5].

Work by Hartwig and Gumplinger [6] used Geant4 to simulate the output pulse shapes from EJ-309, a PSD-capable liquid scintillator developed by Eljen Technologies that has three known decay time components. The first two decay time components were simulated. When compared to experimental data, the simulated pulse shapes did not accurately model the rise times, however the decay tail of the pulses was accurately reproduced. Additionally, the influence of the timing response of the photodetector chosen to convert scintillation photons into an electrical output was not included in the simulation. This is a potential source of inaccuracy when comparing simulated pulse shapes to experimental data.

The pulse shapes derived from inorganic scintillation detectors have been simulated by Ogawara and Ishikawa [7] using a combination of the optical photon tracking capabilities of Geant4 and the experimental response function of a photomultiplier tube (PMT). The arrival time distribution produced by Geant4 to represent the light pulse shape was convoluted with the experimentally measured pulse shape from the PMT to produce the final simulated pulses. While the simulated pulses compared favourably to experimental measurements, the method used to capture the PMT response was identified as a possible source of inaccuracy. This is because the response included the influence of not only the PMT but also the response of a plastic scintillator with fast timing properties that was used in the experimental set-up to obtain the PMT response function.

\section{GEANT4 PULSE SHAPE MODELLING}

Geant4 is an object-oriented C++ Monte Carlo toolkit for simulating the passage of particles through matter [5]. Particles and their interactions can be tracked through arbitrarily complex geometries, material compositions and electromagnetic fields. Geant4 is widely used for the simulation of organic and inorganic scintillation detectors. This is due to its ability to handle optical physics processes, including scintillation and optical photon transportation. Scintillator materials are characterised by their light yield (photons/MeV), photon emission spectrum and the distribution of emission times. These must be defined by the user when constructing the simulation.

An EJ-276 PSD-capable plastic scintillator coupled to a PMT has been simulated using Geant 4 version 10.7. A cube and slab geometry have been constructed to enable a comparison of the pulse shapes from two different geometries and investigate the capabilities of Geant 4 to model more than two decay time components. The cube and slab scintillator geometries are shown in Fig. 1a and Fig. 2a, respectively. The location of the photocathode of the PMT is outlined in red. The light yield was set to 8,600 photons/MeV for neutron and gamma radiation. All three decay time components and their relative weightings for neutron and gamma radiation were input into the simulation, utilising the new enhanced time constant method available in Geant4 10.7. This allows for the specification of more than two decay time components for both particle-independent and particle-dependent yields. Experimental values obtained by Iwanowska-Hanke et al. [8] have been used for the three decay time components and their relative weightings for neutron and gamma radiation.

The Geant4 physics list was constructed using the QGSP_BIC_HP_EMZ reference physics list. This contains QGSP_BIC_HP for hadronic interactions and G4EmStandardPhysics_option4 for electromagnetic interactions. G4OpticalPhysics was included for the generation and transportation of scintillation photons.

Geant 4 has been used for the generation and transportation of individual scintillation photons up to their detection at the photocathode of the PMT. Once detected, their time of arrival was recorded and histogrammed to produce the simulated neutron and gamma ray pulses shown in Fig. $1 \mathrm{~b}$ and Fig. $2 \mathrm{~b}$. These pulses preclude the influence of the timing response of the PMT and any associated data electronics on the shape of the pulse. For each geometry, 10,000 events were simulated using a monoenergetic source of neutron and gamma radiation with an energy of $2 \mathrm{MeV}$.

\section{RESULTS}

The average simulated pulse shapes for an EJ-276 plastic scintillation detector with a cube geometry is shown in Fig. 1b for neutron (blue) and gamma (red) radiation. The pulses have been normalised to enable comparison between neutron and gamma pulses.

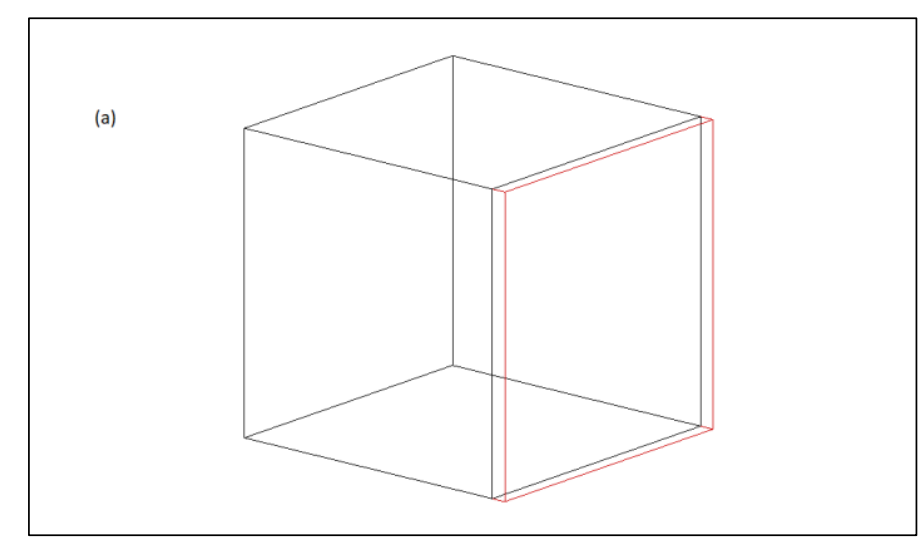




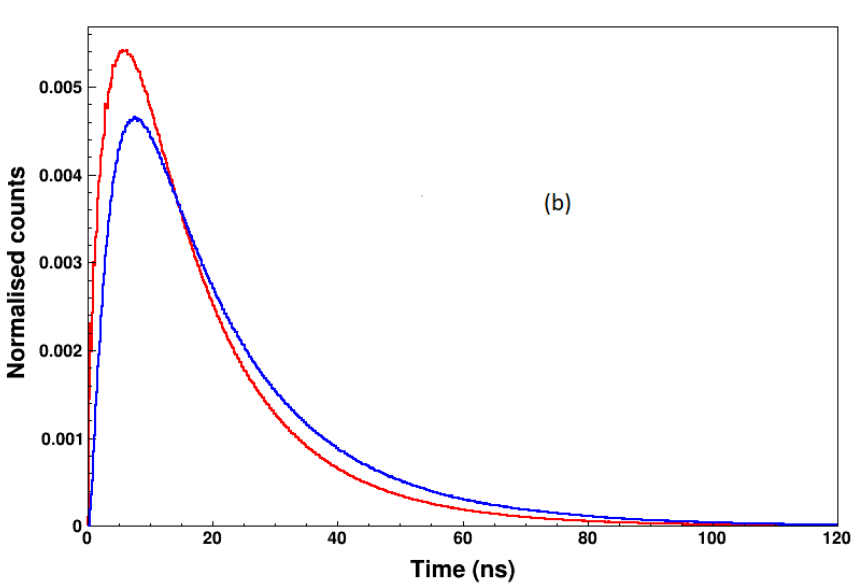

Fig 1. Simulated cube geometry (a) and average output pulse shapes (b) for neutron (blue) and gamma (red) radiation. The dimensions of the cube are $5 \mathrm{~cm}$ x $5 \mathrm{~cm} \times 5 \mathrm{~cm}$.

The pulses simulated approximate those that are observed experimentally for an organic scintillation detector; however, an experimental comparison is required to quantify the accuracy of the simulations. Fig. 1b demonstrates the capability of Geant4 to simulate the important features of the pulses derived from an organic scintillation detector; the rising edge and decay tail. The decay tail is the crucial component for distinguishing particle type. Fig. $1 \mathrm{~b}$ shows the expected differences between neutron and gamma ray induced pulse shapes. The enhanced slow component is observed in the decay tail of the neutron pulse from approximately $20 \mathrm{~ns}$, demonstrating the subtle differences expected between the pulses generated as a result of the interaction of different particles. This forms the basis of PSD and demonstrates the ability of Geant 4 to correctly simulate the expected differences in pulse shape for neutron and gamma radiation using the new enhanced time constant method available in Geant4 version 10.7 .

(a)

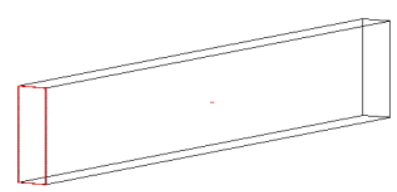

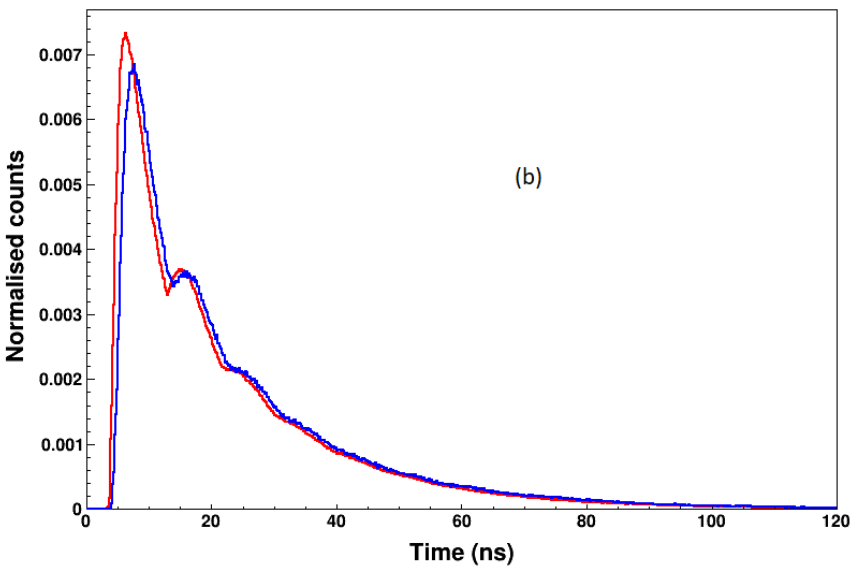

Fig 2. Simulated slab geometry (a) and average output pulse shapes (b) for neutron (blue) and gamma (red) radiation. The dimensions of the slab are $5 \mathrm{~cm}$ x $15 \mathrm{~cm}$ x $100 \mathrm{~cm}$.

For a comparison between two different geometries, a slab geometry was simulated using the same source and number of events. This is shown in Fig. 2b. Compared with the pulse shapes generated for the cube geometry, the differences between the neutron and gamma pulse shapes are small. There is a peak at $10 \mathrm{~ns}$ and a secondary peak at approximately $15 \mathrm{~ns}$ for both pulses. This secondary peak is not visible for the cube geometry. This secondary peak is likely the result of optical photons travelling longer distances and experiencing more reflections in the scintillator before being detected at the photocathode. The poorer separation of pulses for the slab geometry, particularly in the decay tail of the pulse, will result in the slab having a reduced PSD performance compared to the cube geometry. This is expected and agrees with previous studies [3] that observed a dependence of PSD performance on geometry.

\section{FUTURE WORK}

Future work will focus on improving the pulse shape simulations with the aim of reproducing experimental pulse shapes output from an EJ-276 plastic scintillation detector. While Geant 4 can successfully model the temporal pulse shapes from PSD-capable plastic scintillation detectors, they do not provide an accurate representation of that which would be measured experimentally. This is because Geant 4 is only capable of outputting the arrival time distribution of scintillation photons at the photodetector and not the final output pulse shape. This is influenced by not only the scintillation emission but also the complex processes occurring in the photodetector.

Experimental work is planned to measure the temporal pulse shape of an ET-Enterprises 9214 PMT, independent of the properties of the scintillator. This will be done using a nanosecond pulsed laser with a wavelength of $425 \mathrm{~nm}$, matching the peak emission spectrum of EJ-276. The PMT response will be integrated with existing simulations to obtain the final simulated pulse shape. This will enable PSD to be performed on the simulated pulse shapes and comparisons to be made with experimental data. The effect of the PMT response on the overall shape of the pulses can also be quantified. 
Simplifications have been made to the pulse shape simulations to allow for an investigation into the influence of the various factors which affect the shape of the pulses. In order to replicate experimental pulses, optical surface effects and materials used for coupling the PMT to the scintillator will need to be included.

\section{CONCLUSIONS}

This paper demonstrates the capability of Geant 4 version 10.7 to model the temporal pulse shapes output from a PSDcapable plastic scintillation detector with three known decay time components. The expected differences between neutron and gamma pulse shapes due to the way the different particles lose energy in the scintillator are visible, primarily in the decay tail of the pulses. Two different geometries have been compared to show how the pulse shapes change with geometrical configuration. Future work will integrate an experimentally obtained PMT temporal response with existing pulse shape simulations with the aim of accurately reproducing experimental data. The ability to accurately simulate the pulse shapes from organic scintillation detectors allows for the possibility to assess the PSD performance of such detectors in the early stages of detector development.

\section{REFERENCES}

[1] F.D. Brooks "Development of Organic Scintillators", Nuclear Instruments and Methods, VOL. 162 477-505, 1979.

[2] C. Payne, P. L. Sellin, M. Ellis, K. Duroe, et al., Neutron/gamma pulse shape discrimination in EJ-299-34 at high flux. 2015 IEEE Nuclear Science Symposium and Medical Imaging Conference. pp.1-5. 7581994.

[3] Hubbard, Michael W. J, "Light transport modelling of pulse shape discrimination within plastic scintillators. Doctoral thesis, University of Surrey.

[4] Comrie, A., Buffler, A., Smit, F. D. \& Wortche, H. J. Tests of pulse shape discrimination with EJ299-33 plastic scintillator for use in portable spectroscopy in Proceedings, 3rd International Conference on Technology and Instrumentation in Particle Physics (TIPP 2014): Amsterdam, Netherlands, June 2-6, 2014 TIPP2014 (2015), 251. doi:10.22323/1.213.0251.

[5] S. C. J. Allison, K. Amako, J. Apostolakis, H. Araujo, P. Arce Dubois, M. Asai, G. Barrand, R. Capra et al., "Geant4 Developments and Applications," IEEE Trans. Nucl. Sci., vol. 53, no. 1, pp. 270-278, 2006.

[6] Z. S. Hartwig and P. Gumplinger, "Simulating response functions and pulse shape discrimination for organic scintillation detectors with Geant4," Nucl. Instruments Methods Phys. Res. Sect. A Accel. Spectrometers, Detect. Assoc. Equip., vol. 737, pp. 155-162, 2014.

[7] R. Ogawara and M. Ishikawa, "Signal Pulse Emulation for Scintillation Detectors using GEANT4 Monte Carlo with light tracking simulation," VOL., 075114, No. July, 2016.

[8] Iwanowska-Hanke, J et al. Comparative study of large samples (2"2") plastic scintillators and EJ309 liquid with pulse shape discrimination (PSD) capabilities. Journal of Instrumentation 9, P06014 (2014). 